\title{
High power experimental studies of hybrid photonic band gap accelerator structures
}

\author{
JieXi Zhang, ${ }^{*}$ Brian J. Munroe, Haoran Xu, Michael A. Shapiro, and Richard J. Temkin \\ Plasma Science and Fusion Center, Massachusetts Institute of Technology, \\ Cambridge, Massachusetts 02139, USA
}

(Received 3 February 2016; published 31 August 2016)

\begin{abstract}
This paper reports the first high power tests of hybrid photonic band gap (PBG) accelerator structures. Three hybrid PBG (HPBG) structures were designed, built and tested at $17.14 \mathrm{GHz}$. Each structure had a triangular lattice array with 60 inner sapphire rods and 24 outer copper rods sandwiched between copper disks. The dielectric PBG band gap map allows the unique feature of overmoded operation in a $\mathrm{TM}_{02}$ mode, with suppression of both lower order modes, such as the $\mathrm{TM}_{11}$ mode, as well as higher order modes. The use of sapphire rods, which have negligible dielectric loss, required inclusion of the dielectric birefringence in the design. The three structures were designed to sequentially reduce the peak surface electric field. Simulations showed relatively high surface fields at the triple point as well as in any gaps between components in the clamped assembly. The third structure used sapphire rods with small pin extensions at each end and obtained the highest gradient of $19 \mathrm{MV} / \mathrm{m}$, corresponding to a surface electric field of $78 \mathrm{MV} / \mathrm{m}$, with a breakdown probability of $5 \times 10^{-1}$ per pulse per meter for a 100 -ns input power pulse. Operation at a gradient above $20 \mathrm{MV} / \mathrm{m}$ led to runaway breakdowns with extensive light emission and eventual damage. For all three structures, multipactor light emission was observed at gradients well below the breakdown threshold. This research indicated that multipactor triggered at the triple point limited the operational gradient of the hybrid structure.
\end{abstract}

DOI: 10.1103/PhysRevAccelBeams.19.081304

\section{INTRODUCTION}

High gradient accelerator cavities are designed ideally to support only the accelerating mode and suppress the higher-order modes (HOMs). The excitation of HOMs may lead to longitudinal and transverse emittance dilution or the beam breakup, and eventually beam losses in a cavity. One novel method to damp HOMs is to use a photonic band gap (PBG) cavity. Photonic band gap (PBG) accelerator structures have the attractive feature that they are designed to support a single electromagnetic mode, thus reducing or eliminating unwanted modes generated by wakefields in an accelerator [1]. The two-dimensional (2D) periodic photonic crystal array can be constructed by arranging elements of metallic or dielectric materials in the transverse direction. By removing a few central elements of the periodic structure, a "defect" can be formed to confine electromagnetic fields [1-3]. During the past decades, experimental and theoretical studies on PBG structures have been conducted involving room temperature metallic materials and superconducting materials, as well as dielectric materials [4-14].

\footnotetext{
zhangjx03@gmail.com

Published by the American Physical Society under the terms of the Creative Commons Attribution 3.0 License. Further distribution of this work must maintain attribution to the author $(s)$ and the published article's title, journal citation, and DOI.
}

Previous research has demonstrated the confinement of the accelerating mode. A six-cell traveling wave (TW) metallic PBG (MPBG) structure was built and successfully applied to accelerate electrons at a gradient of $35 \mathrm{MV} / \mathrm{m}$ with a $\mathrm{TM}_{01}$ mode [4]. Follow-on experimental research was carried out to investigate the wakefield damping properties of the metallic PBG structure [15]. In order to achieve higher gradient, new standing wave PBG structures were designed to put into high power testing. Metallic PBG structures were high power tested to investigate breakdown phenomena at SLAC at X-Band, $11.424 \mathrm{GHz}$, and at MIT at $\mathrm{Ku}-\mathrm{Band}, 17.14 \mathrm{GHz}[11,12,16,17]$. The gradients and breakdown probabilities achieved by the metallic PBG structures were found to be comparable with those of conventional disk-loaded waveguide (DLWG) cavities $[16,17]$. Wakefield damping has been studied in simulation for metallic PBG structures including both room temperature cavities and superconducting cavities [13,18]. A superconducting PBG cavity for damping high order modes in a superconducting structure has recently been successfully tested [19].

Conventional cavities use the $\mathrm{TM}_{01}$ mode as the operation mode for acceleration, which makes damping the most dangerous HOM, the $\mathrm{TM}_{11}$ dipole mode a big challenge. Due to the cutoff frequency in the band gap map, the metallic PBG structure used the $\mathrm{TM}_{01}$ mode as the accelerating mode [20]. The same as the conventional 
cavities, the dangerous $\mathrm{TM}_{11}$ dipole mode could be weakly confined in a metallic PBG structure in which only a finite number of rows of rods are included. A dielectric PBG lattice, such as the lattice of sapphire rods described in this paper, has a band gap map without a cutoff frequency [3]. This makes a dielectric PBG cavity fundamentally different from a metallic PBG cavity. Furthermore, the dielectric PBG cavity does not have to operate in the $\mathrm{TM}_{01}$ mode. Operation in a higher order mode allows the potential use of larger irises and larger structure sizes, which may be very important as the accelerator frequency increases. We have developed a specific geometrical design in which the $\mathrm{TM}_{02}$ mode is confined as the accelerating mode [21,22]. The unwanted $\mathrm{TM}_{11}$ dipole mode is located at lower frequency, below the band gap, and is not confined. Although a lattice made up entirely of dielectric rods could be constructed, adding metallic rods in the outside row, where the field strength is low, can enhance the structure $Q[9,22]$. This PBG structure that employs both metallic and dielectric material is called a hybrid photonic band gap (HPBG) structure. Dielectric materials have the potential to increase the structure $Q$ since dielectric loss can be very small. In addition, lack of experimental data about breakdown phenomena on dielectric materials at Ku-Band motivated this high power testing of the HPBG cavity.

This paper reports the design and the first high power tests of HPBG structures. Three structures, the HPBG-d3, the HPBG-d025, and the HPBG-Pin structure, each with a different dielectric rod design, have been high power tested. The latter two structures were designed to sequentially reduce the surface electric field. The dielectric band gap map will be presented in Sec. II, as well as the cavity modes confined by the HPBG structure. Designs of the HPBG-d3 and HPBG-Pin structure will be described in detail in Sec. III. Section IV covers the cold test results, including the demonstration of the overmoded operation. Section V discusses the results of the high power testing of the HPBG structures. Discussion and conclusion are presented in Sec. VI.

\section{DIELECTRIC BAND GAP MAPS AND 2D MODES}

Ceramic and sapphire were two options for our HPBG structure. Due to the ultralow dielectric loss, sapphire rods were chosen. Sapphire is an anisotropic crystal whose dielectric constant varies due to the polarization and the propagation direction of the incident light with respect to the optical axis (C-axis) of the crystal. Orientation of the $\mathrm{C}$-axis parallel to the longitudinal accelerating axis $z$ yields a permittivity of $\varepsilon_{r}=[9.398,9.398,11.587]$ at $17 \mathrm{GHz}$ [23]. The dielectric loss varies with direction, too, as $\tan \delta=[3,3,8.6] \times 10^{-5}$.

The periodic triangular lattice array is formed by a few hexagonal rows of the dielectric (sapphire) rods in the

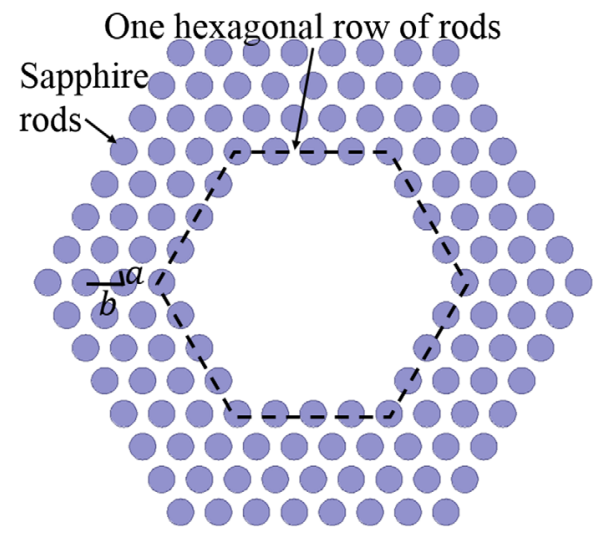

FIG. 1. A 2D, 4-row, dielectric PBG lattice array. The inner 37 rods have been omitted to form the cavity. The rod radius is $a$ and the rod spacing is $b$.

transverse $x y$ plane. The lattice array is sandwiched between two metal plates. The first four hexagonal rows of sapphire rods (thirty-seven rods in total) are removed to form the defect cavity. Figure 1 shows a 2D lattice array model built in the High Frequency Structural Simulator (HFSS) code [24] with four hexagonal rows of sapphire rods. Here $a$ is the radius of the sapphire rod and $b$ is the rod spacing.

Calculating the dispersion relation of the triangular PBG lattice formed by sapphire rods for a series of values of the lattice ratio $a / b$, we obtain the band gap map of sapphire plotted as the normalized frequency $(\omega b / 2 \pi c, c$ is the speed of light) vs $a / b$, as shown in Fig. 2 in yellow. The yellow shaded area indicates the region where band gaps exist. In contrast with the metallic PBG structure, the dielectric band gap map has no cutoff frequency, that is, the lowest frequency modes can propagate in the lattice and are therefore not confined. By setting the frequency of the $\mathrm{TM}_{01}$ mode and the $\mathrm{TM}_{11}$ mode below the first band gap, the $\mathrm{TM}_{02}$ mode can be used as the accelerating mode; this constitutes overmoded operation.

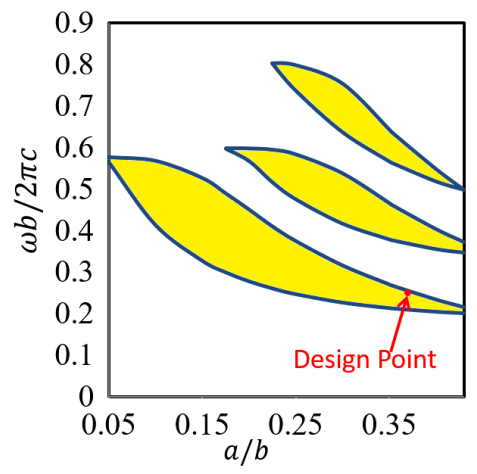

FIG. 2. TM mode band gap map of a 2D lattice of sapphire rods of radius $a$ and spacing $b$. 


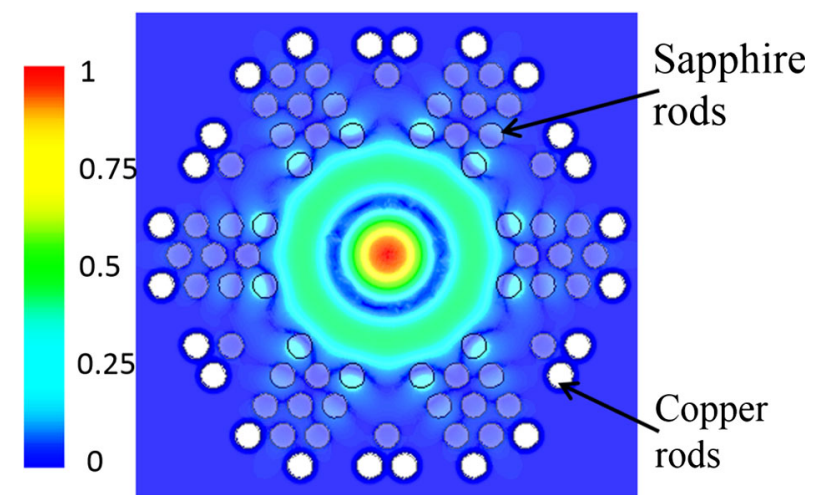

FIG. 3. The electric field of the $\mathrm{TM}_{02}$ mode confined by the HPBG structure.

With the structure shown in Fig. 1, all of the confined TM eigenmodes were calculated, resulting in a design point shown in Fig. 2. At $a / b \sim 0.35$ the $\mathrm{TM}_{02}$ mode sits within the lowest band gap with a high radiative (diffractive) quality factor $Q_{\text {rad }}$. Simulation of the structure showed that there was no confinement of the $\mathrm{TM}_{11}$ mode, which is a significant advantage of the dielectric PBG structure. To achieve $Q_{\text {rad }} \geq 10^{3}$ of the $\mathrm{TM}_{02}$ mode with the concept shown in Fig. 1 would require at least five hexagonal rows of sapphire rods (in total 180 rods). We used three or four hexagonal rows, compensating for the reduction in $Q_{\mathrm{rad}}$ of the $\mathrm{TM}_{02}$ mode by two means: (1) adding one row of copper rods outside the sapphire rods [9]; and (2) removing some rods to make the $\mathrm{TM}_{02}$ mode more azimuthally uniform as well as creating damping channels for some unwanted modes. The new configuration of the hybrid PBG structure is shown in Fig. 3 with the $\mathrm{TM}_{02}$ mode. Table I shows the comparison of the radiative (diffractive) quality factor $Q_{\mathrm{rad}}$ of the $\mathrm{TM}_{02}$ mode between the four-row dielectric PBG cavity and the four-row HPBG cavity, which shows a huge improvement in the HPBG structure. Reference [5] showed that the optimization of the arrangement of the rod pattern could potentially increase $Q_{\text {rad }}$ of the accelerating mode by more than eighty times in a cavity containing only two rows of sapphire rods.

The results for a 2D structure are important for identifying the design point. A detailed three-dimensional (3D) design using the configuration in Fig. 3 for the breakdown experiment based on the HPBG model is described in the following section.

TABLE I. Comparison of radiative $Q$ between the dielectric and the hybrid PBG structures with four rows of rods.

\begin{tabular}{lc}
\hline \hline Structure & $Q_{\text {rad }}$ \\
\hline Dielectric PBG & 710 \\
Hybrid PBG & 24000 \\
\hline \hline
\end{tabular}

\section{3D DESIGN OF THE HPBG STRUCTURE}

To conveniently and comparably test the high power properties of a specific cavity, SLAC developed a standard procedure of testing a single-cell, standing wave (SW) cavity with two side DLWG cavities to confine the high field only in the central test cavity [25-29]. A mode launcher is used to convert the $\mathrm{TE}_{10}$ mode of rectangular waveguide into the $\mathrm{TM}_{01}$ mode of circular waveguide, which can couple directly into the SW test structure. The PBG structures tested at MIT followed the same design concept with a clamped assembly [30] and scaled dimensions to match the resonant frequency of $17.14 \mathrm{GHz}$ of the Haimson Research Corporation (HRC) klystron. Figure 4 shows the 3D model of the HPBG structure used in the SW test stand and Fig. 5 shows all the components of the first HPBG structure used for the high power testing. All parts were clamped together

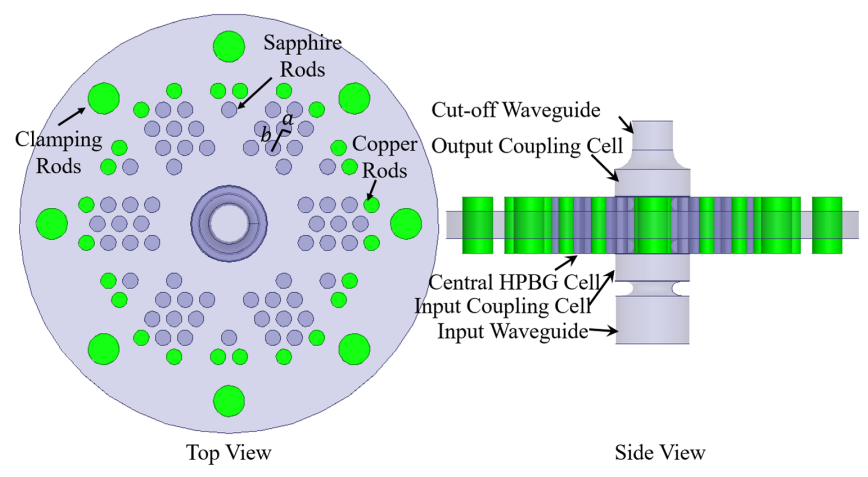

FIG. 4. The model of the HPBG structure as a central cell sandwiched between conventional input and output cylindrical coupling cells in the 3D simulation.

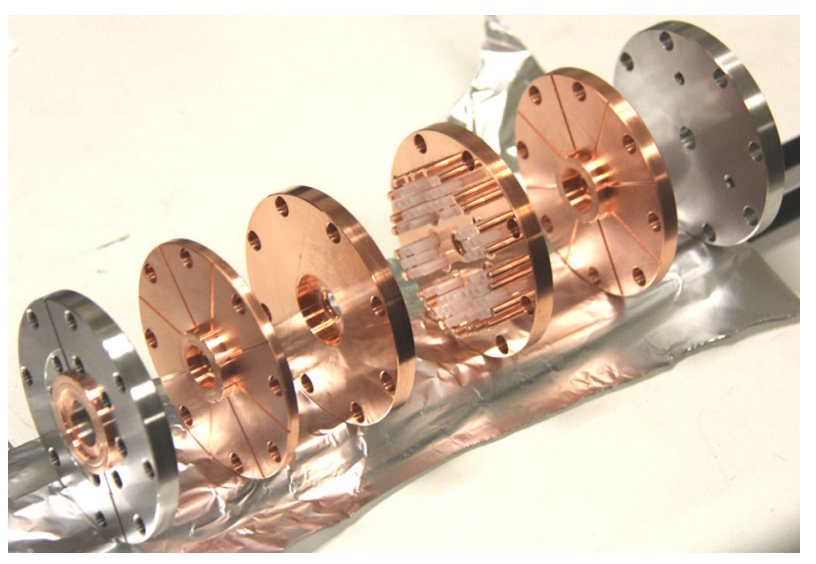

FIG. 5. Machined components of the HPBG structure, including (left to right) the input flange with a copper gasket; the input coupling cell; the upstream HPBG cell plate; the downstream HPBG cell plate with 60 sapphire rods and 20 copper rods inserted; the output coupling cell; and the output flange. The flanges are made of stainless steel and the others are OFHC copper. 


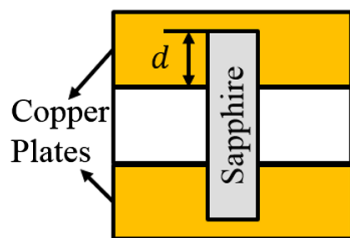

(a) d3 (b) $\mathrm{d} 025$

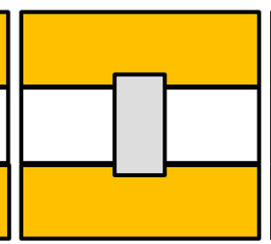

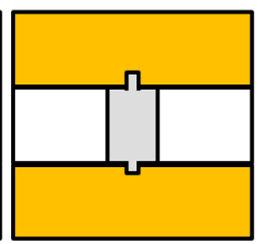

(c) Pin
FIG. 6. Schematic comparison of the three HPBG structures.

by eight clamping rods. The clamped assembly allowed a vector network analyzer (VNA) measurement to be applied to each cell, based on which independent corrections could be applied to each cell separately.

The insertion length $d$ of the sapphire rods into the copper plates was critical in determining the ratio of the highest surface field to the accelerating gradient. To prioritize the mechanical stability, the first HPBG structure employed an insertion length of $d=3 \mathrm{~mm}$ of the sapphire rods into the copper plates, as shown in Fig. 6(a), which we refer to as the HPBG-d3 structure. This structure had the highest ratio of surface electric field to the accelerating gradient. One cause of the high surface fields is the triple point enhancement to the surface fields [31]. The triple point occurs at the location where the dielectric rod is inserted into the metal plate; the triple point is an interface of dielectric, metal and vacuum. The triple point enhancement is a well-known problem of utilization of dielectric material. Surface field can be enhanced by a large factor approaching a triple point. To reduce the strength of the surface $E$ and $H$ fields, a fillet design was applied to the HPBG-d3 structure, which will be discussed in detail in III A 2. And later, to lower the surface fields even more, the HPBG-d025 structure $(d=0.25 \mathrm{~mm})$ and the HPBG-Pin structure were designed sequentially with different sapphire rod shapes, as shown in Figs. 6(b) and 6(c), respectively. In this section, the design of the HPBG-d3 structure and the HPBG-Pin structure will be discussed in detail. The design of the HPBG-d025 structure is similar to that of the HPBG-d3 structure and will be omitted for brevity.

\section{A. HPBG-d3}

\section{Design parameters}

Figure 7 shows the side view of the model of the standing wave (SW), HPBG-d3 structure with the identification of the key parameters with the values listed in Table II.

Among those parameters, the radius of the input waveguide $\left(R_{\mathrm{WG}}\right)$, the thicknesses $\left(t_{i}\right)$ and the major radii $\left(a_{i}\right)$ of the coupling iris, the input iris and the output iris, the radii of the input and output iris apertures $\left(r_{c}\right)$, and the heights of the three cells $\left(d_{c}\right)$ were adjust to match the microwave frequency of $17.14 \mathrm{GHz}$ ( $\pi$ mode). The cutoff end section was rounded off by $r_{\text {end }}=2 \mathrm{~mm}$ and had a final radius $R_{\text {end }}=4.23 \mathrm{~mm}$, sufficiently small to cut off all modes at 17.14 GHz. The radius to round the input and output cavity

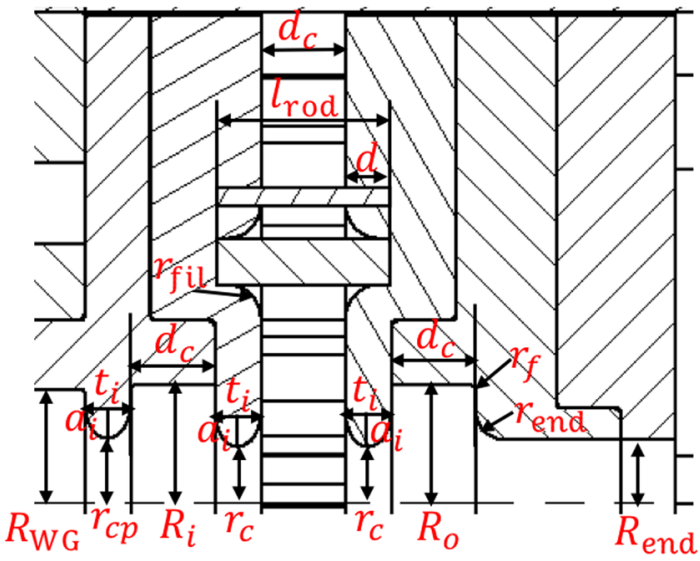

FIG. 7. Parameters specifying the HPBG structure.

edges is $r_{f}=0.25 \mathrm{~mm}$. All the other parameters could be modified to obtain the resonant frequency with a higher quality factor and the minimum surface fields. Adjusting the lattice spacing $b$ combined with the radii of the input $\left(R_{i}\right)$ and the output $\left(R_{o}\right)$ coupling cells could tune the structure. The radius of the coupling aperture, $r_{c p}$ decided the critical coupling. Most importantly, the insertion length $d$, determined the highest surface fields, as described below.

The lattice spacing $b$ in the range $[4.47,4.51] \mathrm{mm}$ gave a resonant frequency close to the klystron frequency with good confinement. A stronger confinement favored a larger radius, leading to $a=1.58 \mathrm{~mm}$ with the design point $a / b \sim 0.35$.

The vacuum wavelength set up the lower limit of the length of a sapphire rod. The thickness of the iris provided an upper limit of the length. In order to stabilize the clamped structure and ensure good alignment, an as-longas-possible insertion length was preferred, leading to $d=3 \mathrm{~mm}$ and $l_{\text {rod }}=11.68 \mathrm{~mm}$. The cylindrical sapphire rods were made by Insaco, Inc.

\section{Insertion length and fillet}

Simulations showed the shorter the insertion length, the lower the value of the maximum surface field. The ideal

TABLE II. Values of the design parameters of the HPBG-d3 structure (unit: $\mathrm{mm}$ ).

\begin{tabular}{lccc}
\hline \hline$a$ & $b$ & $l_{\text {rod }}$ & $d$ \\
\hline 1.58 & 4.48 & 11.68 & 3 \\
\hline$t_{i}$ & $a_{i}$ & $r_{c}$ & $r_{c p}$ \\
\hline 3.07 & 2.27 & 3.765 & 4.33 \\
\hline$R_{\mathrm{WG}}$ & $r_{\text {end }}$ & $R_{\text {end }}$ & $r_{f}$ \\
\hline 7.62 & 2 & 4.23 & 0.25 \\
\hline$R_{o}$ & $R_{i}$ & $r_{\text {fil }}$ & \\
\hline 7.88 & 7.88 & 2.25 & \\
\hline \hline
\end{tabular}




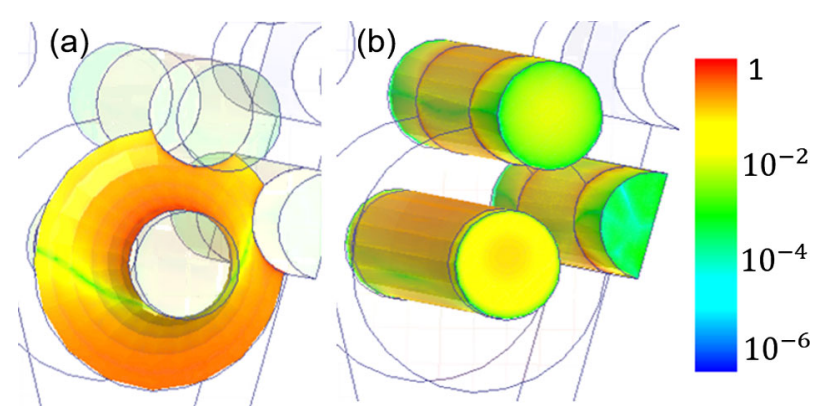

FIG. 8. The normalized electric field map (in log scale) of (a) the fillet, and (b) the inner three rods of the HPBG-d3 structure in simulation.

case of $d=0$ had a maximum surface $E$ field of $E_{\text {surf }} \sim$ $240 \mathrm{MV} / \mathrm{m}$ and an $H$ field of $H_{\text {surf }} \sim 590 \mathrm{kA} / \mathrm{m}$ at $E_{G}=100 \mathrm{MV} / \mathrm{m}$. Those were comparable to a conventional metallic disk-loaded-waveguide (DLWG) cavity [17]. However, the $d=0$ case was not practical. An insertion length of $d=3 \mathrm{~mm}$ increases the maximum values of the surface fields to $E_{\text {surf }} \sim 890 \mathrm{MV} / \mathrm{m}$ and $H_{\text {surf }} \sim 3900 \mathrm{kA} / \mathrm{m}$ at $E_{G}=100 \mathrm{MV} / \mathrm{m}$. In order to reduce the surface fields of the $d=3 \mathrm{~mm}$ structure, a fillet with radius $r_{\text {fil }}=2.25 \mathrm{~mm}$ had to be applied to the holes on the copper plates at the triple-point edge of every innermost rod (12 rods, total). The purpose of the fillet is to provide a rounded surface at this triple point to reduce the strength of the surface $E$ and $H$ fields. Figure 8 shows the surface electric field maps on the fillet and the innermost three rods in relative log scale. Figure 9 shows the top view of the electric and magnetic field maps on the central plane of the HPBG-d3 structure in linear scale. The inclusion of a large fillet migrated the highest surface fields from the triple-point edge of the innermost rod to the edge of the second innermost rod. The surface field distributions are
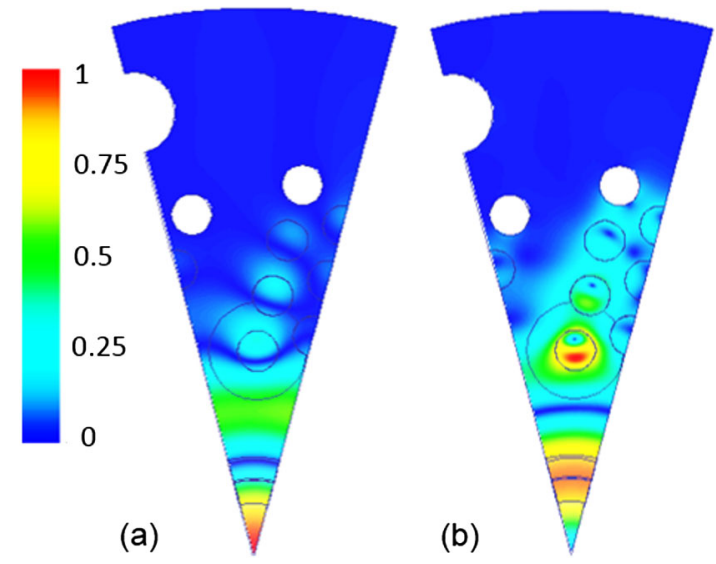

FIG. 9. The normalized field map of (a) the electric field, and (b) the magnetic field of the central plane of the HPBG-d3 structure in simulation. White circles indicate the copper rods, which exclude the fields. The sapphire rod locations are more easily seen in Fig. 10.
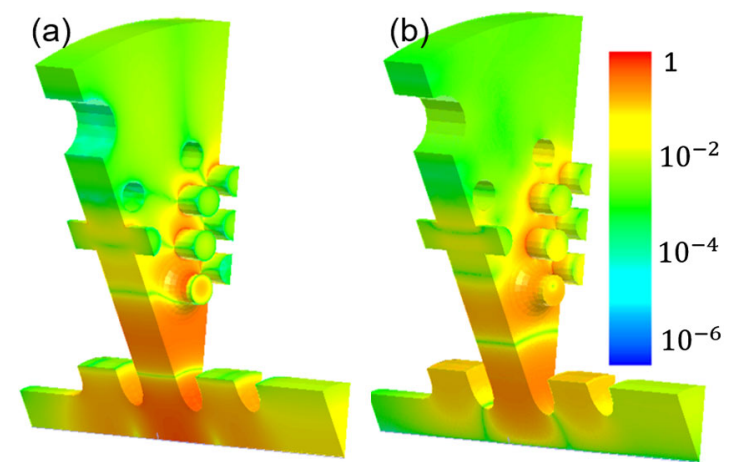

FIG. 10. The normalized field distribution (in log scale) of (a) the surface electric field, and (b) the surface magnetic field of the HPBG-d3 structure in simulation.

shown in Fig. 10. To generate a $100-\mathrm{MV} / \mathrm{m}$ accelerating gradient, the input power required for the SW, HPBG-d3 structure was $11 \mathrm{MW}$, resulting in $E_{\text {surf }}=628 \mathrm{MV} / \mathrm{m}$ (reduced from $\sim 890 \mathrm{MV} / \mathrm{m}$ without the fillet) and $H_{\text {surf }}=2297 \mathrm{kA} / \mathrm{m}$, as listed in Table III with comparison to two other structures. In Table III, $P_{\text {in }}$ is the total power needed for the full SW HPBG structure.

Final adjustments of the three entangled parameters $R_{o}$, $R_{i}, r_{c p}$, and $b$ gave a good theoretical coupling at the right frequency, as shown in Table IV, which also includes a comparison with the other two structures. In Table IV, the loaded $Q, Q_{L}$, is $1 / Q_{L}=1 / Q_{\text {rad }}+1 / Q_{\text {diel }}+1 / Q_{\text {ohm }}$, where $Q_{\text {rad }}$ is the radiative $Q$ of the cavity, including the central PBG cell and the two coupling cells and the radiation through the feeding waveguide, $Q_{\text {diel }}$ represents the absorptive loss in the sapphire rods, and $Q_{\text {ohm }}$ represents the ohmic loss on the copper rods and disks. Among those, the absorptive loss is negligible due to the ultralow dielectric loss of sapphire. The calculated accelerating field profile ( $E_{z}$ on $z$ axis) is shown in Fig. 11, demonstrating that the high field is obtained only in the central HPBG cell.

TABLE III. Comparison of the peak surface $E$ and $H$ fields of the HPBG structures for a $100 \mathrm{MV} / \mathrm{m}$ accelerating gradient.

\begin{tabular}{lccc}
\hline \hline HPBG & $E_{\text {surf }}(\mathrm{MV} / \mathrm{m})$ & $H_{\text {surf }}(\mathrm{kA} / \mathrm{m})$ & $P_{\text {in }}(\mathrm{MW})$ \\
\hline $\mathrm{d} 3$ & 628 & 2297 & 11 \\
$\mathrm{~d} 025$ & 434 & 1335 & 6.5 \\
Pin & 391 & 1016 & 4.5 \\
\hline \hline
\end{tabular}

TABLE IV. Comparison of the $f_{0}$ and $Q$ of the HPBG structures in simulation.

\begin{tabular}{lcc}
\hline \hline HPBG & $f_{0}(\mathrm{GHz})$ & $Q_{L}$ \\
\hline $\mathrm{d} 3$ & 17.1446 & 2230 \\
$\mathrm{~d} 025$ & 17.1366 & 3120 \\
Pin & 17.1399 & 4080 \\
\hline \hline
\end{tabular}




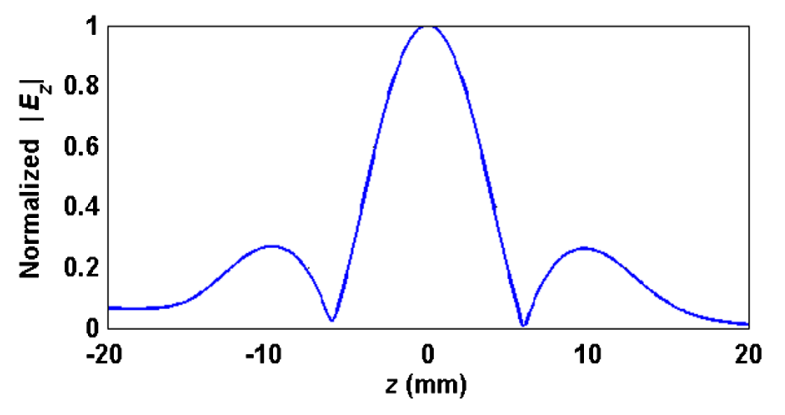

FIG. 11. The normalized magnitude of the electric field on z-axis of the HPBG-d3 structure simulated in HFSS.

\section{B. HPBG-d025}

An HPBG-d025 structure was successfully built and tested. As can be seen from Table III, it is predicted that the surface fields in the HPBG-d025 structure fall in between those of the HPBG-d3 and the HPBG-Pin structures. In fact, this theoretical result was confirmed in cold test and in hot test at high gradient [32]. In the interest of brevity, we will omit a detailed discussion of the results for this structure since the results obtained fall in between those of the other structures.

\section{HPBG-Pin}

This design, as shown in Fig. 6(c), employed a new "rolling-pin" shaped sapphire rod in the innermost two hexagonal rows to lower the high fields at the triple-point. The rolling-pin sapphire rod consists of two parts, a main cylindrical rod (3.16 $\mathrm{mm}$ diameter by $5.68 \mathrm{~mm}$ long) and two small projections (1 $\mathrm{mm}$ diameter by $1 \mathrm{~mm}$ long) extending into the copper plate. Figure 12 shows a top view of the central cell of the HPBG-Pin structure. Figure 13(a) shows a rolling-pin rod built by Gavish, Inc. The configuration in Fig. 12 was designed to minimize the surface electric and magnetic fields. The ideal rolling-pin rod had a straight (right angle) connection between the cylindrical

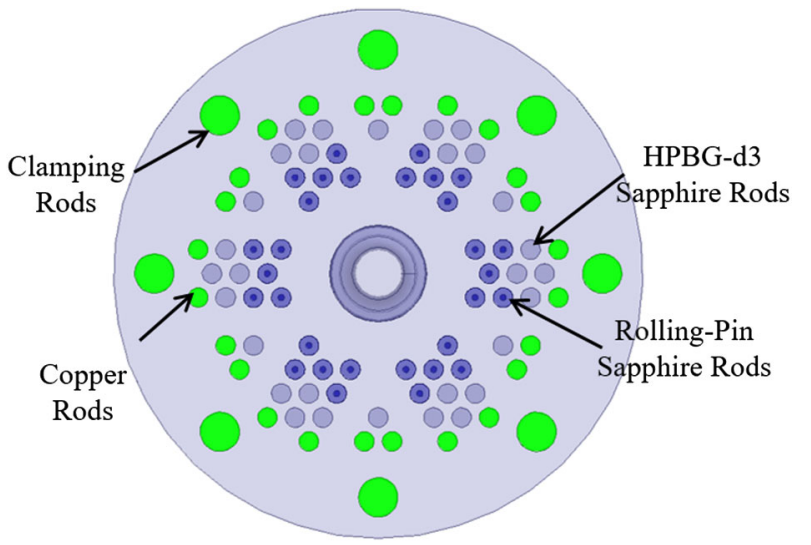

FIG. 12. Top view of the HFSS model of the HPBG-Pin structure. (a)
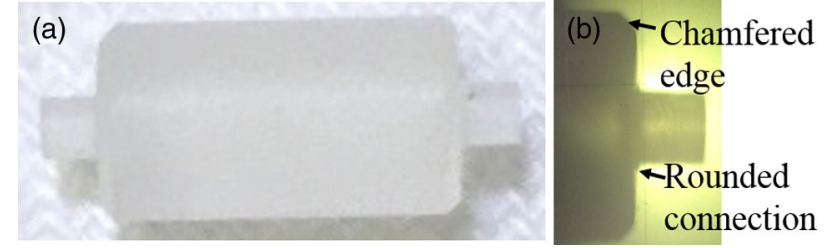

FIG. 13. (a) The rolling-pin sapphire rod made by Gavish, Inc., and (b) a comparator image showing the rounded connection and the chamfered edge.

main part and the pin part, which would lower the maximum value of the surface electric field to $E_{\text {surf }}=$ $220 \mathrm{MV} / \mathrm{m}$ at $E_{G}=100 \mathrm{MV} / \mathrm{m}$. This value of the $E$ field would occur on the iris of the central cell, and not at the rod surface or triple point. Also, in this case, the magnitude and the location of the maximum $E$ field would be the same as in a DLWG and a metallic PBG structure. However, the rolling-pin rods could not be built with such a right angle, but instead had a $0.13-\mathrm{mm}$ radius rounded connection from the main cylinder to the pin and a $0.2-\mathrm{mm}$ radius chamfered edge, as shown in Fig. 13(b). Those changes enhanced the surface electric field. To simplify the assembly of the HPBG-Pin structure, the outer sapphire rods, which see greatly reduced surface fields, were straight cylindrical rods
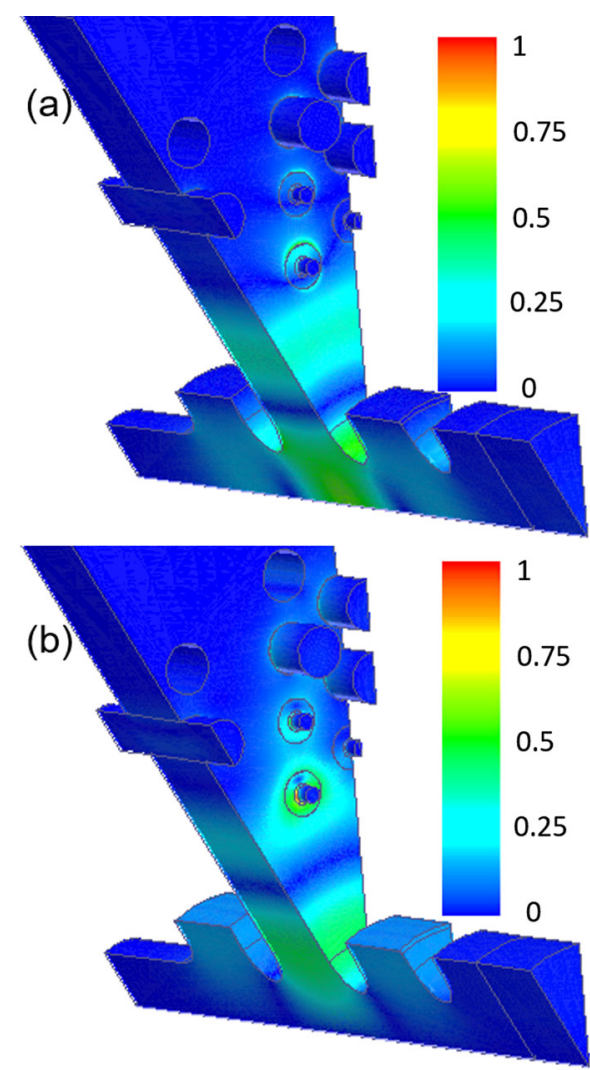

FIG. 14. The field distribution of (a) the surface electric field, and (b) the surface magnetic field of the HPBG-Pin structure in simulation. 
with $d=3 \mathrm{~mm}$ (same as in the HPBG-d3 structure) and the copper rods had $d=1 \mathrm{~mm}$. An HFSS simulation reflecting the as-built shape of the rolling-pin rods is shown in Fig. 14. The peak values, $E_{\text {surf }}=391 \mathrm{MV} / \mathrm{m}$ and $H_{\text {surf }}=$ $1016 \mathrm{kA} / \mathrm{m}$ at a $100 \mathrm{MV} / \mathrm{m}$ gradient, are listed in Table III. The other parameters distinct from the HPBGd3 design were $R_{i}=7.82 \mathrm{~mm}, R_{o}=7.84 \mathrm{~mm}$ and $r_{c p}=$ $3.93 \mathrm{~mm}$ to give good coupling for the HPBG-Pin structure and a higher $Q$. The design results of the 3D HPBG-Pin structure are listed in Tables III and IV.

\section{COLD TEST}

\section{A. $S_{21}$ measurement}

A transmission measurement was designed and applied to the central HPBG cell where the $\mathrm{TM}_{02}$ mode was excited [22]. Figure 15 shows the copper plate with rods inserted and the central cell assembly of the HPBG-d3 structure. All the metallic parts were machined by the MIT Central Machine Shop. An Agilent E8363B Vector Network Analyzer (VNA) was used to measure the $S$ parameters. Two coaxial antennas, each held by a three-axis translation stage, were connected to the VNA to send and receive

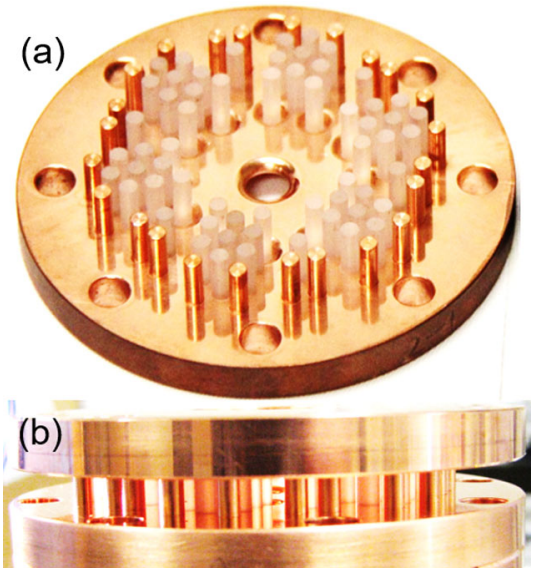

FIG. 15. (a) The rod plate with rods inserted, and (b) the central HPBG cell assembled for the $S_{21}$ measurement.

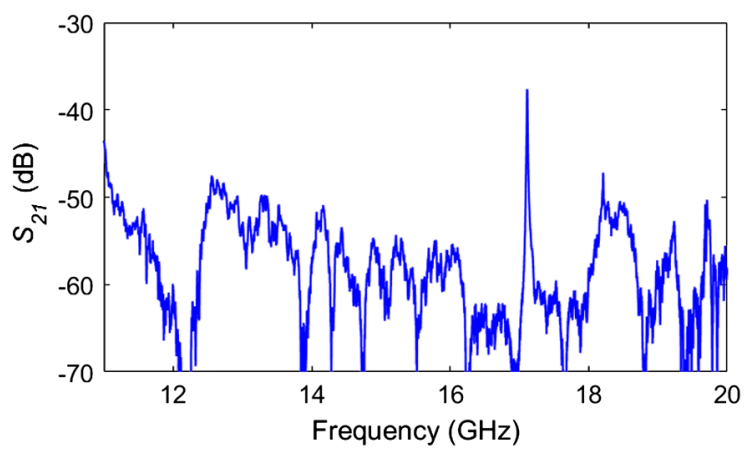

FIG. 16. The magnitude of $S_{21}$ of the HPBG cell. signals. In practice, the antennas were kept at a distance relatively far away from the HPBG cell to minimize the antenna loading of the cavity (thus $Q_{0} \sim Q_{L}$ ) but close enough to receive a good signal-to-noise ratio (SNR) of the measurement.

Figure 16 shows the measured result, with a single dominant resonant frequency $f_{0}=17.113 \mathrm{GHz}$ and a loaded quality factor $Q_{L}=2790$, demonstrating the single, overmoded excitation. A frequency slightly lower than 17.14 GHz comes from the iris-coupling of the wave to free space which resulted in a slightly longer wavelength and thus a lower frequency. Figure 16 indicates that the only resonance is the $\mathrm{TM}_{02}$ mode, showing the potential of the HPBG structure for wakefield damping.

\section{B. Resonant properties by $S_{11}$ measurement}

A calibrated $S_{11}$ measurement of the resonant frequency and the quality factor was applied to the completely assembled HPBG structure. All of the parts were clamped using eight clamping rods and connected to the mode launcher to have the microwave power coupled in, as shown in Fig. 17. If the measurement showed poor coupling, simulations would be performed to modify the

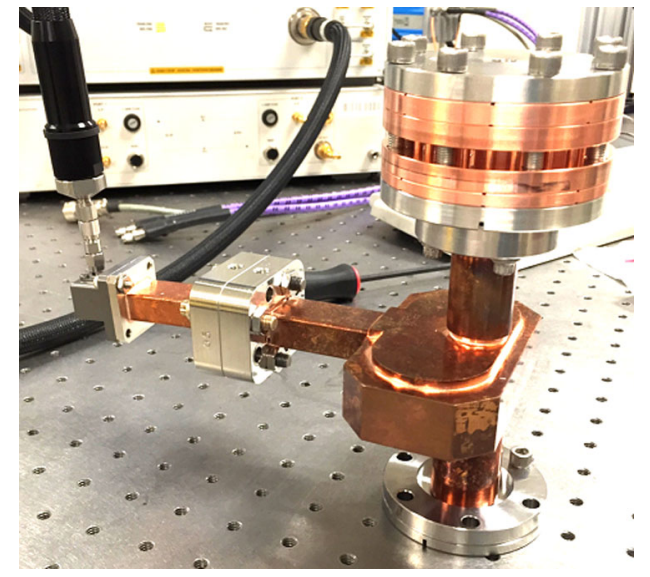

FIG. 17. The clamped HPBG structure with mode launcher under test at the VNA.

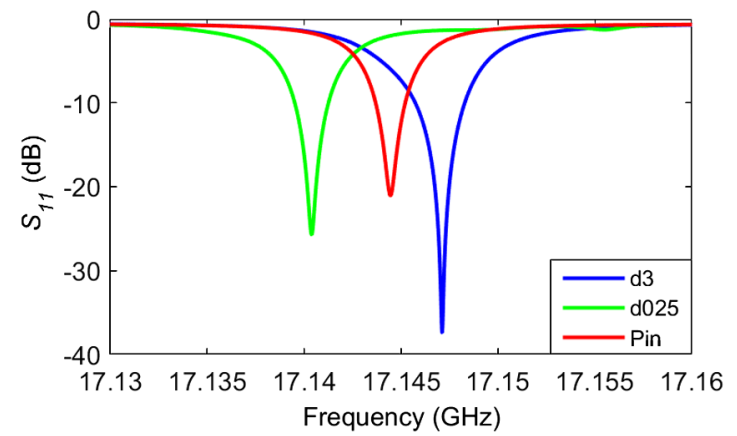

FIG. 18. Results of the $S_{11}$ measurement of the three HPBG structures. 
TABLE V. Comparison of $f_{0}$ and $Q_{L}$ of the three HPBG structures in $S_{11}$ measurement.

\begin{tabular}{lccc}
\hline \hline HPBG & $f_{0}(\mathrm{GHz})$ & $S_{11}(\mathrm{~dB})$ & $Q_{L}$ \\
\hline $\mathrm{d} 3$ & 17.147 & -37 & 2150 \\
d025 & 17.140 & -26 & 2980 \\
Pin & 17.144 & -21 & 3390 \\
\hline \hline
\end{tabular}

sizes of the two coupling cells $\left(R_{i}, R_{o}\right)$ and the aperture of the coupling iris $\left(r_{c p}\right)$. An adjustment of the lattice spacing $b$ could be made if the resonance was not at the right frequency range. When this occurred, a new pair of copper plates with new spacing $b$ was built and cold tested. Usually after two to three iterations, the HPBG structure achieved good coupling at the right frequency. The results of the $S_{11}$ measurements for all three structures are shown in Fig. 18 and Table V. All three structures had very good coupling at the correct frequency $(17.145 \pm 0.01 \mathrm{GHz})$.

\section{Field profile by bead pull measurement}

A resonant perturbation measurement, in the form of a "bead pull" measurement was conducted to measure the accelerating field profile $[33,34]$. In our measurement, the bead was formed by a super glue drop adhering to a thin dielectric wire. The wire, supported on a roller system, was connected to a stepping motor to precisely move the bead up and down. The HPBG structure was bolted to the roller system and aligned so as to make the bead go along the accelerating axis. Due to the small bead size, we could measure the magnitude and the phase change of $S_{11}$ at the resonant frequency caused by the bead perturbing the local field. The bead pull measurements of the three HPBG structures all returned a $\pi$-mode field profile, with the high field only in the central HPBG field. As an example, the result of the HPBG-Pin structure is shown in Fig. 19. The nonzero value of the normalized field at large values of the axial distance, outside of the cavity, is believed to be caused by experimental errors in the bead pull measurement. These errors may arise from the extremely small size of the bead and its instability during the bead pull measurement and from small calibration errors.

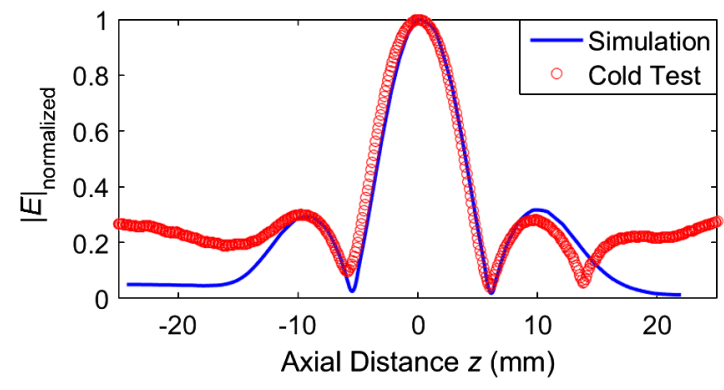

FIG. 19. The axial $E$ field profile of the HPBG-Pin structure by bead pull measurement.

\section{HIGH POWER TESTING}

\section{A. Experimental setup and diagnostics}

The procedure for the high power, high gradient testing of the HPBG structures was similar to what had been conducted with the metallic PBG structures [11,12,16,17]. The experiments were conducted at the MIT PSFC Accelerator Laboratory.

The high power microwave (HPM) source for the breakdown experiment was a relativistic klystron built by Haimson Research Corporation (HRC). It could produce 10-1000 ns square microwave pulses with an output power up to $25 \mathrm{MW}$. The central frequency is $17.145 \mathrm{GHz}$ with a bandwidth of $20 \mathrm{MHz}$. The output power of the HRC klystron was transmitted to the standing wave (SW) test stand for the high power testing. The HPBG structure was put into a vacuum chamber inside of which the pressure was kept at $10^{-8}$ Torr using an ion pump. A 4.4-dB hybrid between the klystron and the test stand reduced the level of power reflected back to the klystron. A ceramic window was used to isolate the vacuum system between the klystron and the test stand. The maximum rf power transmitted to the SW test stand was $3.63 \mathrm{MW}$.

Figure 20 schematically shows the diagnostics used for the breakdown experiment of the HPBG structures. The power coupled into the HPBG cell could be calculated from the incident (forward) power signal. Comparing with the simulation, the gradient, the surface electric and magnetic fields, and the temperature rise [35] could be obtained. The reverse signal was used to check if the microwave frequency was at the right resonant frequency. A single highdirectivity waveguide directional coupler was connected to the waveguide to monitor the forward and the reverse microwave power. The connection was located close to the test stand to ensure an accurate detection. The forward and the reflected microwave power were detected using Hewlett

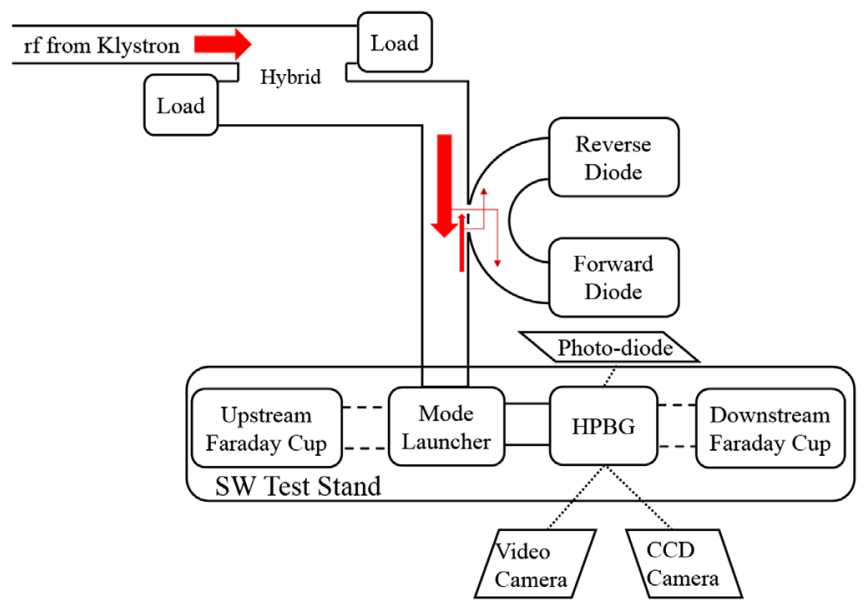

FIG. 20. Diagnostics used for the high power testing of the HPBG structures. 
Packard 8473B low-barrier Schottky diodes. The diode signals were coupled into an Agilent Infiniium DSO-S 404A High-Definition Oscilloscope.

Two copper Faraday cups were attached to the HPBG structure to monitor the dark current (DC) generated on each shot. The downstream Faraday cup was bolted to the output flange of the HPBG structure. The upstream Faraday cup was bolted to the other end of the mode launcher. Both Faraday cups were electrically isolated from the HPBG structure. Each Faraday cup was connected to a BNC cable with a $50-\Omega$ load to couple the current signal to two channels on the same oscilloscope. Breakdown events could be determined by the reverse power signal and the dark current signals.

The open PBG cavity made a direct optical diagnostic possible. A video camera connected to a cathode ray tube display was used to visually observe the real-time light flash generated in the HPBG cell. A Thorlabs 8050M-GE 8 Megapixel Monochrome Scientific Charge-Coupled Device (CCD) Camera was used to capture the light in the HPBG cell. A Thorlabs silicon Avalanche Photodiode APD130A2 was attached to the window of the SW test stand to measure the amount of light emitted by the structure on every shot.

\section{B. Experimental results of the HPBG-d3 structure}

The first high power tests of the HPBG-d3 structure included 458,000 high power shots. A preliminary description of these results was reported in Ref. [36]. In this subsection, we include a more complete description, allowing comparison to results from our other tested structures. Figure 21 shows the summary of the gradient and the magnitude of the dark current of each input shot.

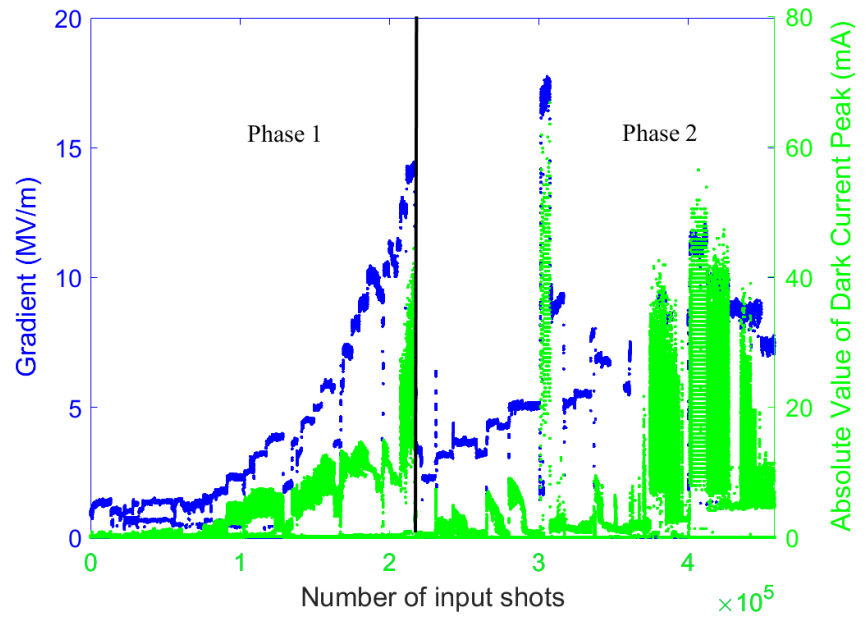

FIG. 21. The high power tests of the HPBG-d3 structure of gradient (blue) and the magnitude of the dark current (green) from the downstream Faraday cup.

\section{Processing}

The structure testing was conducted in two phases. In Phase 1, the structure experienced processing, with relatively higher dark current than that of Phase 2 at the same gradient level. The two phases are shown in Fig. 21, with a vertical line dividing the phases.

The input pulse was kept at $100 \mathrm{~ns}$ in Phase 1 . Breakdown started to happen on every shot at $E_{G} \geq 3 \mathrm{MV} / \mathrm{m}$. The HPBG-d3 structure was greatly processed after $\sim 222,000$ shots, indicated by much lower dark current values at the same gradient.

After processing, Phase 2 included 236,000 shots. In Phase 2, for each new gradient level, the structure needed $\sim 10^{4}$ shots to be reprocessed. During the reprocessing, the dark current decreased gradually. After reprocessing, the dark current stayed at an order of magnitude lower than in Phase 1 . The structure came to a quasi-steady state with a constant breakdown probability. In Phase 2 , the pulse length of the input shot was varied from 100 ns to $160 \mathrm{~ns}$ to result in an approximately 80-ns flat top of the calculated structure power, as shown in Fig. 22. Figure 22 shows the traces of the non breakdown pulse and the breakdown pulse in Phase 2. Here the structure power and the gradient were calculated from the forward power signal without considering the reflected power or the absorption by multipactor. Therefore, the structure power was only accurate for shots without breakdown. Breakdown was determined when a reflection was observed on the reverse channel with a higher dark current.

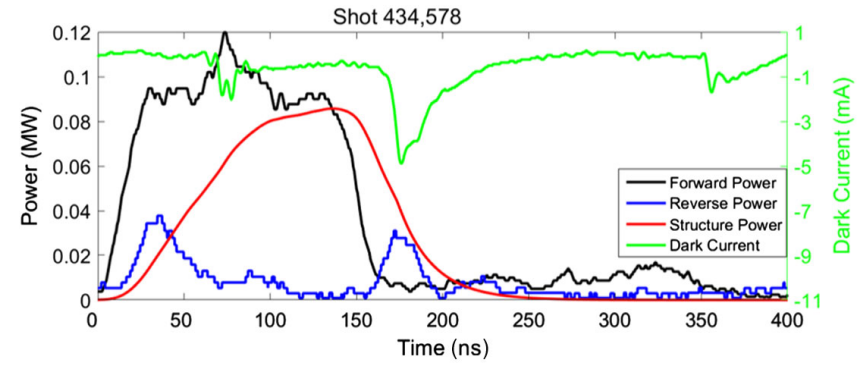

(a) $E_{G}=8.55 \mathrm{MV} / \mathrm{m}$, no breakdown Shot 434,628

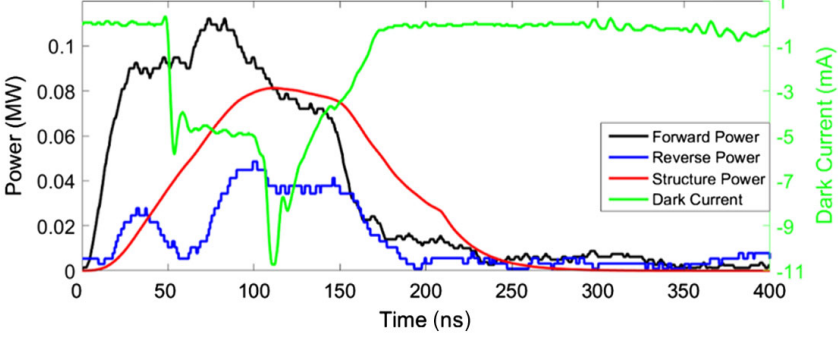

(b) $E_{G}=8.33 \mathrm{MV} / \mathrm{m}$. breakdown

FIG. 22. Typical traces of the forward power, the reverse power, the calculated structure power and the dark current from the downstream Faraday cup for the HPBG-d3 structure with (a) no breakdown, and (b) breakdown showing the increased reverse power and dark current during breakdown. 


\section{Dark current and light emission}

Comparing with the metallic PBG structure tested at MIT [16,17], the dark current of the HPBG-d3 structure was much higher even at a low gradient level. Two sources may be responsible: (1) the high surface $E$ field which was enhanced by the triple point [31] and the gaps between the components introduced by the clamped assembly, and (2) multipactor which was more easily triggered by a system including a dielectric. The dark current always rose at a delay time after the beginning of the input power, as shown in the traces in Fig. 22(a). The delay time decreases monotonically as the gradient increases. The range of the delay times is about $50-80 \mathrm{~ns}$, which was consistent with previous observations of the buildup of multipactor and electron avalanche [37].

Figure 23(a) shows a side view of the central hybrid cell of the HPBG-d3 structure held in the test chamber taken through a viewport in ambient light. Clamping rods, copper rods and sapphire rods are visible. During the experiment, the test chamber was covered by a black curtain so that only a few copper rods could be seen with background light, as shown in Fig. 23(b). Figure 23(c) shows light emission on sapphire rods (indicated by arrows).

There was light emission at $E_{G}>5.7 \mathrm{MV} / \mathrm{m}$ on every shot with or without breakdown. This was evidence of plasma formation along the sapphire rods due to multipactor. At increasing gradient, the multipactor can trigger electron avalanche, plasma formation, surface material outgassing and eventually breakdown. The onset of multipactor discharges at dielectric surfaces exposed to high electric field strengths has been studied extensively theoretically [38-42]. Previous experimental research on dielectric accelerator structures at X-Band has reported the onset of multipactor effects at gradients in the $\mathrm{MV} / \mathrm{m}$ range [43-47]. The present experiments have the additional factor of having a triple point at the location where the rods enter the supporting copper plates [31]. The deleterious effects of microwave-induced breakdown at the triple point have been extensively studied, for example, in window breakdown research $[37,48,49]$.

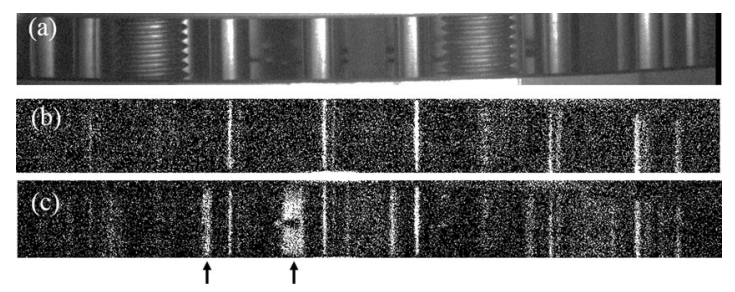

FIG. 23. CCD images of the central cell of the HPBG-d3 structure of (a) ambient light view showing sapphire, copper and clamping rods, (b) background light illuminating the cooper rods on a low gradient shot without breakdown, and (c) light emission on the sapphire rods (indicated by arrows) at $E_{G}>5.7 \mathrm{MV} / \mathrm{m}$. This view is similar to the view shown in Fig. 15(b).

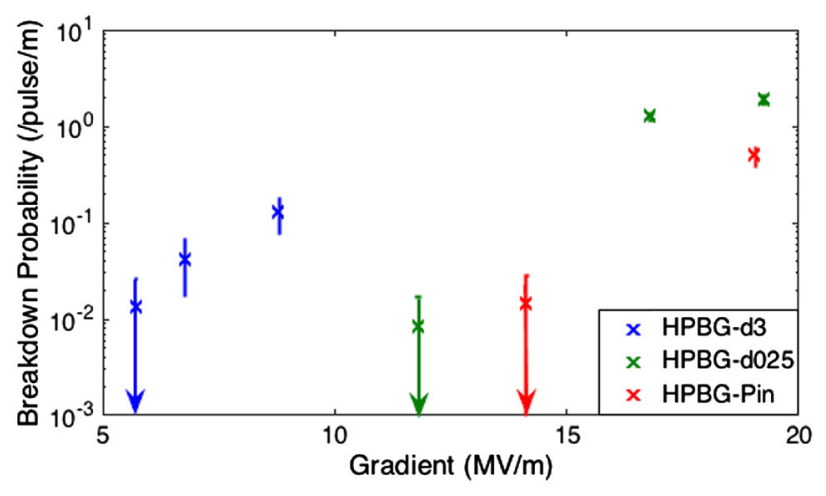

FIG. 24. Breakdown probability as a function of gradient of the three HPBG structures.

\section{Breakdown probability}

The breakdown probability of the HPBG-d3 structure after reprocessing is plotted as a function of gradient in Fig. 24. The data of the HPBG-d3 structure are for 160-ns input pulses. The error bar of the breakdown probability was calculated statistically based on the Poisson distribution. For the HPBG-d3 structure, at $E_{G}=5.7 \mathrm{MV} / \mathrm{m}$, no breakdowns were observed in two hours' running of about $8.6 \times 10^{3}$ shots. For clarity, a single breakdown event was assigned, resulting in an error bar of the same value, which was indicated by an arrow going down to zero in the log scale plot. The highest gradient achieved for HPBG-d3 in 160 -ns input pulse testing was $8.8 \mathrm{MV} / \mathrm{m}$, with a breakdown probability of $1.28 \times 10^{-1}$ per pulse per meter. Increasing the gradient to $9.6 \mathrm{MV} / \mathrm{m}$ resulted in a runaway condition with chains of breakdowns, an order of magnitude higher dark current, and no sign of conditioning after $\sim 2.7 \times 10^{4}$ input shots.

\section{Post-test}

The post-test cold test showed a small reduction of the quality factor $Q$, as shown in Table VI, with comparison to the HPBG-d3 structure before the high power testing and the structure with the same metallic plates after testing but a new set of sapphire rods. The small reduction in the loaded $Q$ after test was believed to be the result of damage to the sapphire rods.

Due to the clamped design, the structure could be disassembled without suffering any additional damage.

TABLE VI. Comparison of the resonant frequency $f_{0}$ and the quality factor $Q_{L}$ of the HPBG-d3 structure before and after the high power testing.

\begin{tabular}{lcc}
\hline \hline & $f_{0}(\mathrm{GHz})$ & $Q_{L}$ \\
\hline Before Test & 17.147 & 2150 \\
After Test & 17.149 & 2010 \\
After, with New Sapphire Rods & 17.141 & 2170 \\
\hline \hline
\end{tabular}




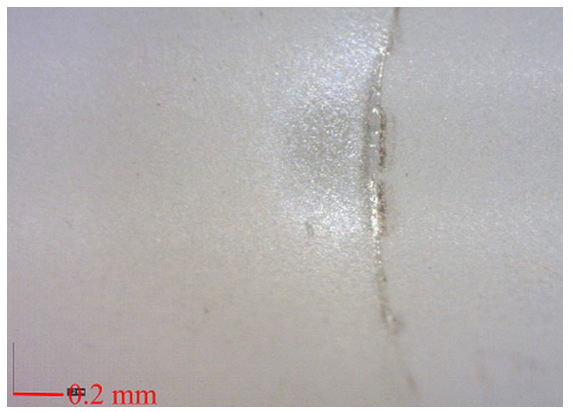

FIG. 25. Microscope image showing breakdown damage at the triple-point edge of the sapphire rod.

Simulations gave the highest field occurring at the triplepoint edge of the second innermost rod, where damage was in fact found on the metallic plate and on some of the sapphire rods. On the surface of one sapphire rod, there was some erosion and copper deposition, as shown in Fig. 25.

\section{Experimental results of HPBG-Pin}

The high power tests of the HPBG-Pin structure lasted for $\sim 150,000$ shots at $17.14 \mathrm{GHz}$. Figure 26 shows the summarized results. Data from shots numbered $5.32 \times 10^{4}$ to $6.32 \times 10^{4}$ were lost, as shown by a gap in the data in Fig. 26, but the lost data do not affect the conclusions.

\section{Processing and light emission}

Based on the high power tests of the foregoing two HPBG structures, much higher power (corresponding to $E_{G}=25 \mathrm{MV} / \mathrm{m}$ ) was put into the HPBG-Pin structure at the beginning phase in order to fast-condition the structure. The dark current of this stage was just a single sharp negative spike, proportional to gradient, similar to its two antecedents. In the processing phase, the structure started to break down on every shot at a low gradient level $(\sim 5 \mathrm{MV} / \mathrm{m})$. In order to facilitate the conditioning progress, the gradient level was raised to an even higher

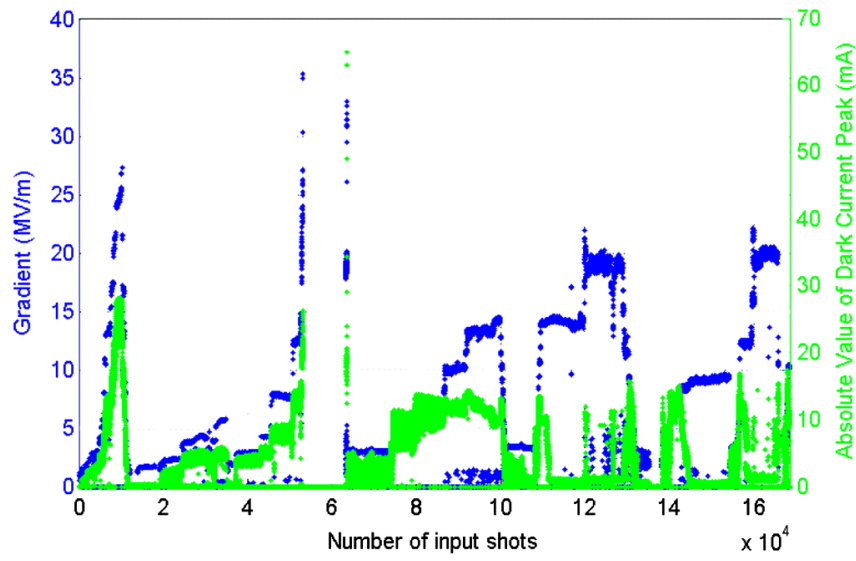

FIG. 26. The high power testing of the HPBG-Pin structure for gradient (blue) and the magnitude of the dark current (green). level $(\sim 35 \mathrm{MV} / \mathrm{m})$. Even at this high power, the structure held steady with acceptable dark current $(\sim 30 \mathrm{~mA})$ and no severe breakdowns for $\sim 1 \times 10^{4}$ shots. The CCD camera captured some highlight flashes. After a few shots, the captured light started to dim and fade away. This situation lasted for about three thousand shots, until a few runaway events occurred in which many rods emitted light. At that time, the input power was lowered immediately and the structure processing and testing were continued.

The processing started to show effectiveness after around $9 \times 10^{4}$ shots, resulting in lower and lower dark current at the same gradient. The CCD camera showed light emission at $E_{G} \geq 9.3 \mathrm{MV} / \mathrm{m}$, which was likely due to multipactor discharges. As the gradient was increased, about two thousand shots were needed to reprocess the structure. Figures 27(a) and 27(b) compare the traces during and after the reprocessing at a similar gradient. The high spike of the dark current in the beginning was gradually smoothed out, leaving only the main part. Breakdown was determined when the spike of the dark current occurred again after reprocessing, as shown in Fig. 27(a). In Fig. 27, the shape of the structure power calculated is close to triangular and

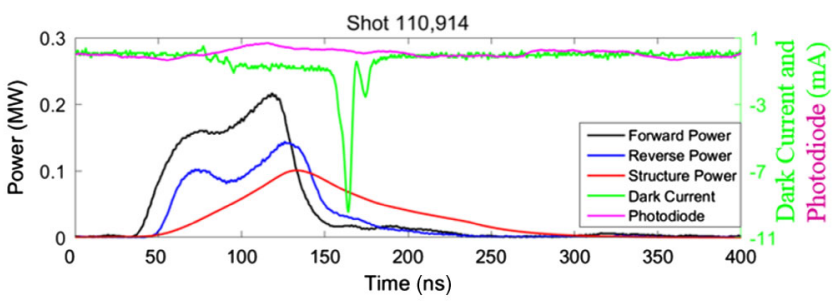

(a) $E_{G}=14.1 \mathrm{MV} / \mathrm{m}$, breakdown Shot 116,574

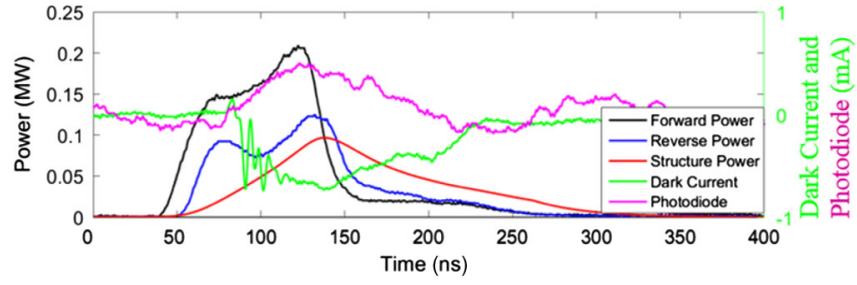

(b) $E_{G}=13.8 \mathrm{MV} / \mathrm{m}$, no breakdown Shot 165,627

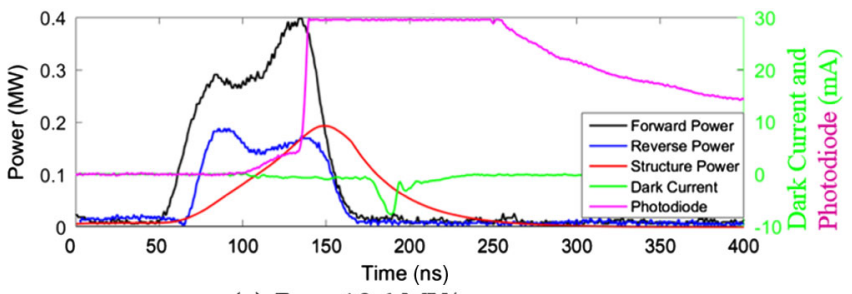

(c) $E_{G}=19.6 \mathrm{MV} / \mathrm{m}$, runaway

FIG. 27. Typical traces of the forward power, the reverse power, the calculated structure power and the dark current from the downstream Faraday cup for the HPBG-Pin structure (a) during reprocessing, (b) after reprocessing, no breakdown, and (c) for runaway situation of the high power testing of the HPBG-Pin structure. 


\section{II IIII IIIIIIII}

FIG. 28. CCD image of the central cell of the HPBG-Pin structure taken on a severe breakdown shot during the high power testing.

does not have a flat top. This came from the short pulse length (100 ns) of the input power.

The gradient was raised to $19.1 \mathrm{MV} / \mathrm{m}$, where a good measurement was made of the breakdown probability. A further increase in gradient to $E_{G} \sim 20 \mathrm{MV} / \mathrm{m}$ resulted in severe breakdowns, with saturation of the photodiode. A similar runaway situation happened to the HPBG-d025 structure at $E_{G} \sim 20 \mathrm{MV} / \mathrm{m}$. Both structures could not recover even at much lower gradients. Figure 27(c) shows the saturation of the photodiode and Fig. 28 shows the breakdown light explosion filling the cavity space. A pressure rise of the ion pump connected to the vacuum chamber was observed, indicating outgassing from the HPBG-Pin structure.

\section{Breakdown probability of the HPBG-Pin structures}

The breakdown probability vs gradient of the HPBG-Pin structure is shown in Fig. 24. The highest operational gradient achieved for the HPBG-Pin structure was $19.1 \mathrm{MV} / \mathrm{m}$, with a breakdown probability of $5.0 \times 10^{-1}$ per pulse per meter for a 100-ns input power pulse.

\section{Post-test}

The cold test results after the high power experiment of the HPBG-Pin structure indicated no degradation in $Q$. However, the autopsy showed damage on the rounded connection between the pin part and the main part of the rolling-pin rods. In addition to similar erosion and copper deposition on the sapphire rods, a more severe crack happened on one rod, as shown in Fig. 29. The damage to the rods may have been caused by the enhancement of the $E$ field at the inevitable small gaps between the sapphire rods and the copper plates in the clamped structure.

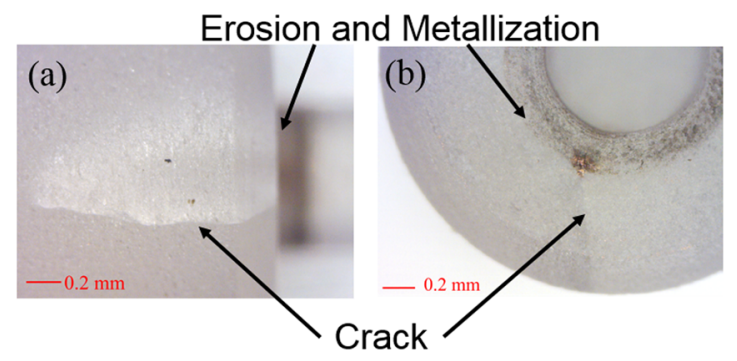

FIG. 29. (a) The side view, and (b) the top view of the images of the crack on the rolling-pin sapphire rod.

\section{DISCUSSION AND CONCLUSIONS}

This paper reports the first high power tests of a hybrid photonic band gap (HPBG) accelerator structure. The results were obtained with three overmoded accelerator structures operating at $17.14 \mathrm{GHz}$.

Operation of the HPBG structure in the $\mathrm{TM}_{02}$ mode does reduce the shunt impedance compared with a comparable $\mathrm{TM}_{01}$ mode structure. The $\mathrm{TM}_{02}$ mode does have the advantage of allowing a larger cavity size, which is an advantage in operation at a very high frequency. The use of very low loss sapphire rods leads to a high value of loaded $Q$ when compared with an all-metal structure, resulting in less power dissipation and less stored energy.

The highest operational gradient achieved in the hybrid PBG structure was $19.1 \mathrm{MV} / \mathrm{m}$ with a breakdown probability of $5.0 \times 10^{-1}$ per pulse per meter for a 100 -ns input power pulse. $19 \mathrm{MV} / \mathrm{m}$ is well below the gradient achieved with metallic structures but, to our knowledge, is the highest gradient achieved with a dielectric structure. The limit on the achievable gradient of the HPBG structures results from the high surface $E$ field which is in addition enhanced at the gaps between components in the clamped assembly. Besides, inclusion of a dielectric makes the system vulnerable to multipactor, especially at the triple point, leading to breakdown. For both the HPBG-d025 and the HPBG-Pin structure, a runaway situation occurred at a gradient at or above $20 \mathrm{MV} / \mathrm{m}$. A few severe breakdowns happened with saturation on the photodiode and extensive light emission. Those two structures could not recover even at much lower gradients, marking permanent damage in the hybrid cavities. Light emitted from the sapphire rods was observed for all of the three hybrid structures on every shot with a gradient higher than a threshold corresponding to a surface $E$ field of $\sim 32-36 \mathrm{MV} / \mathrm{m}$. For the HPBG-Pin structure, this corresponded to $E_{G} \sim 9.3 \mathrm{MV} / \mathrm{m}$, way below the breakdown threshold. The light emission indicated the emergence of multipactor and plasma formation. Breakdown damage was found at the triple point interface where the peak surface electric field was located, or the greatest field enhancement occurred. Discoloration and erosion were observed on both the sapphire rods and the copper plates. Metallization of copper onto the surface of the dielectric rods could potentially degrade the performance and the quality of the hybrid structure, and thus may be a disadvantage of the hybrid design.

These high power breakdown experiments have revealed the role of multipactor and the triple point in triggering breakdown and limiting the operational gradient for a hybrid accelerator structure containing metal and dielectric components. For each hybrid structure, the dark current value after processing could be much lower than before, indicating the conditioning progress to reduce the multipactor effect. Rapid processing is applicable to the dielectric accelerator cavity. Using the results of the current hybrid PBG structure testing, an optimized hybrid structure 
design could be developed. To get to a higher gradient, a brazed structure with metal-coated rod tips should be applied to avoid the enhancement on surface fields caused by the gaps at the triple points. In addition, titanium nitride could be coated on the surface of the sapphire rods to help suppress multipactor [46]. In fact, the ceramic/dielectric rods used in a hybrid photonic structure may suffer from charging by secondary particles generated by a high intensity beam. These particles may have sufficient energy to penetrate into the rods through a coating, such as titanium nitride, applied to the rods. Low-loss ceramics having finite dc conductivity are being developed in order to resolve this problem, but the rf losses of such materials compared to sapphire may be an issue, especially at high rf frequencies.

\section{ACKNOWLEDGMENTS}

This work was supported by the Department of Energy High Energy Physics, under Grant No. DE-SC0010075. The authors would like to thank Mr. Ivan Mastovsky for engineering support in the accelerator laboratory, Dr. Sami Tantawi for supplying the mode launcher and Dr. Jake Haimson for helpful comments.

[1] N. Kroll, D. R. Smith, and S. Schultz, Photonic band gap structures: A new approach to accelerator cavities, AIP Conf. Proc. 279, 197 (1992).

[2] E. Yablonovitch, Inhibited Spontaneous Emission in Solid-State Physics and Electronics, Phys. Rev. Lett. 58, 2059 (1987).

[3] J. D. Joannopoulos, S. G. Johnson, J. N. Winn, and R. D. Meade, Photonic Crystals: Molding the Flow of Light, 2nd ed. (Princeton University Press, New Jersey, 2008), ISBN 9780691124568.

[4] E. I. Smirnova, A. S. Kesar, I. Mastovsky, M. A Shapiro, and R.J. Temkin, Demonstration of a 17-GHz, HighGradient Accelerator with a Photonic-Band-Gap Structure, Phys. Rev. Lett. 95, 074801 (2005).

[5] C. A. Bauer, G. R. Werner, and J. Cary, Truncated photonic crystal cavities with optimized mode confinement, J. Appl. Phys. 104, 053107 (2008).

[6] G. R. Werner, C. A. Bauer, and J. R. Cary, Wakefields in photonic crystal cavities, Phys. Rev. ST Accel. Beams 12, 071301 (2009).

[7] M. R. Masullo, A. Andreone, E. D. Gennaro, S. Albanese, F. Francomacaro, M. Panniello, V. G. Vaccaro, and G. Lamura, Study of hybrid photonic band gap resonators for particle accelerators, Microwave Opt. Technol. Lett. 48, 2486 (2006).

[8] E. D. Gennaro, S. Savo, A. Andreone, V. Galdi, G. Castaldi, V. Pierro, and M. R. Masullo, Mode confinement in photonic quasicrystal point-defect cavities for particle accelerators, Appl. Phys. Lett. 93, 164102 (2008).

[9] E. D. Gennaro, C. Zannini, S. Savo, A. A, M. R. Masullo, G. Castaldi, I. Gallina, and V. Galdi, Hybrid photonicbandgap accelerating cavities, New J. Phys. 11, 113022 (2009).
[10] C. Jing, F. Gao, S. Antipov, Z. Yusof, M. Conde, J. G. Power, P. Xu, S. Zheng, H. Chen, C. Tang, and W. Gai, Observation of wakefields in a beam-driven photonic band gap accelerating structure, Phys. Rev. ST Accel. Beams 12, 121302 (2009).

[11] R. A. Marsh, M. A. Shapiro, R. J. Temkin, V. A. Dolgashev, L. L. Laurent, J. R. Lewandowski, A. D. Yeremian, and S. G. Tantawi, X-band photonic band-gap accelerator structure breakdown experiment, Phys. Rev. ST Accel. Beams 14, 021301 (2011).

[12] B. J. Munroe, A. M. Cook, M. A. Shapiro, R. J. Temkin, V. A. Dolgashev, L. L. Laurent, J. R. Lewandowski, A. D. Yeremian, S. G. Tantawi, and R. A. Marsh, High power breakdown testing of a photonic band-gap accelerator structure with elliptical rods, Phys. Rev. ST Accel. Beams 16, 012005 (2013).

[13] E. I. Simakov, S. S. Kurennoy, J. F. OHara, E. R. Olivas, and D. Y. Shchegolkov, Optimizing the configuration of a superconducting photonic band gap accelerator cavity to increase the maximum achievable gradients, Phys. Rev. ST Accel. Beams 17, 022001 (2014).

[14] C. A. Bauer, G. R. Werner, and J. R. Cary, Origin and reduction of wakefields in photonic crystal accelerator cavities, Phys. Rev. ST Accel. Beams 17, 051301 (2014).

[15] R. A. Marsh, Experimental Study of Photonic Band Gap Accelerator Structures, Ph.D. thesis, MIT, 2009.

[16] B. J. Munroe, Experimental studies of novel accelerator structures at $11 \mathrm{GHz}$ and $17 \mathrm{GHz}$, Ph.D. thesis, MIT, 2015.

[17] B. J. Munroe, J. X. Zhang, H. Xu, M. A. Shapiro, and R. J. Temkin, Experimental high gradient testing of a $17.1 \mathrm{GHz}$ photonic band-gap accelerator structure, Phys. Rev. Accel. Beams 19, 031301 (2016).

[18] M. Hu, B. J. Munroe, M. A. Shapiro, and R. J. Temkin, Calculation of wakefields in a $17 \mathrm{GHz}$ beam-driven photonic band-gap accelerator structure, Phys. Rev. ST Accel. Beams 16, 022002 (2013).

[19] S. Arsenyev, W. B. Haynes, D. Shchegolkov, E. Simakov, and T. Tajima, High Gradient Testing of the Five-cell Superconducting RF Module with a PBG Coupler Cell, in Proceedings of the 6th International Particle Accelerator Conference (JACoW, Richmond, Virginia, 2015), WEPTY082, pp. 3471-3474, http://jacow.org/IPAC2015/ papers/wepty082.pdf.

[20] E. I. Smirnova, C. Chen, M. A. Shapiro, J. R. Sirigiri, and R. J. Temkin, Simulation of photonic band gaps in metal rod lattices for microwave applications, J. Appl. Phys. 91, 960 (2002).

[21] M. A. Shapiro, E. I. Smirnova, C. Chen, and R. Temkin, Theoretical analysis of overmoded dielectric photonic band gap structures for accelerator applications, in Proceedings of the 2003 Particle Accelerator Conference, Portland, OR (IEEE, New York, 2003), Vol. 2, p. 1255, http://accelconf .web.cern.ch/AccelConf/p03/PAPERS/TPAB028.PDF.

[22] J. X. Zhang, A. M. Cook, B. J. Munroe, M. A. Shapiro, and R. J. Temkin, Design and cold test of a $17 \mathrm{GHz}$ overmoded hybrid PBG accelerator cavity, in Proceedings of the 25th Particle Accelerator Conference, PAC2013, Pasadena, CA, 2013 (IEEE, New York, 2013), p. 357, http://accelconf.web.cern.ch/accelconf/pac2013/ papers/mopsm06.pdf. 
[23] J. Krupka, K. Derzakowski, M. Tobar, J. Hartnett, and R. G. Geyer, Complex permittivity of some ultralow loss dielectric crystals at cryogenictemperatures, Meas. Sci. Technol. 10, 387 (1999).

[24] High Frequency Structure Simulator, Ansys, Inc. http:// www.ansys.com/.

[25] V. Dolgashev, S. Tantawi, C. Nantista, Y. Higashi, and T. Higo, Travelling wave and standing wave single cell high gradient tests, in Proceedings of the 2004 Linear Accelerator Conference, Lubeck, Germany, 2004 (DESY, Hamburg, 2004), pp. 766-768, http://slac.stanford.edu/ cgi-wrap/getdoc/slac-pub-10667.pdf.

[26] V. A. Dolgashev, S. G. Tantawi, C. D. Nantista, Y. Higashi, and T. Higo, RF breakdown in normal conducting singlecell structures, in Proceedings of the 21st Particle Accelerator Conference, Knoxville, TN, 2005 (IEEE, Piscataway, NJ, 2005), p. 595, http://accelconf.web.cern.ch/AccelConf/ p05/PAPERS/ROAC007.PDF.

[27] V. A. Dolgashev, S. G. Tantawi, Y. Higashi, and B. Spataro, Study of basic RF breakdown phenomena in high gradient vacuum structures, in Proceedings of the 25th International Linear Accelerator Conference, LINAC2010, Tsukuba, Japan (KEK, Tsukuba, Japan, 2010), p. 1043, https://accelconf.web.cern.ch/accelconf/ LINAC2010/papers/fr105.pdf.

[28] V. A. Dolgashev, S. G. Tantawi, A. Yeremian, Y. Higashi, and B. Spataro, Status of high power tests of normal conducting single-cell standing wave structures, in Proceedings of the International Particle Accelerator Conference, Kyoto, Japan (ICR, Kyoto, 2010), pp. 3810, http://accelconf.web.cern.ch/AccelConf/IPAC10/papers/ thpea060.pdf.

[29] V. A. Dolgashev, S. Tantawi, Y. Higashi, and B. Spataro, Geometric dependence of radio-frequency breakdown in normal conducting accelerating structures, Appl. Phys. Lett. 97, 171501 (2010).

[30] B. Spataro, D. Alesini, V. Chimenti, V. A. Dolgashev, Y. Higashi, M. Migliorati, A. Mostacci, R. Parodi, S. G. Tantawi, and A. Yeremian, High-power comparison among brazed, clamped and electroformed X-band cavities, Nucl. Instrum. Methods Phys. Res., Sect. A 657, 88 (2011).

[31] N. M. Jordan, Y. Y. Lau, D. M. French, R. M. Gilgenbach, and P. Pengvanich, Electric field and electron orbits near a triple point, J. Appl. Phys. 102, 033301 (2007).

[32] J. X. Zhang, Experimental studies of hybrid photonic band gap accelerator structures, Ph.D. thesis, MIT, 2016.

[33] L. C. Maier and J. C. Slater, Field strength measurements in resonant cavities, J. Appl. Phys. 23, 68 (1952).

[34] C. W. Steele, A nonresonant perturbation theory, IEEE Trans. Microwave Theory Tech. 14, 70 (1966).

[35] D. P. Pritzkau and R. H. Siemann, Experimental study of rf pulsed heating on oxygen free electronic copper, Phys. Rev. ST Accel. Beams 5, 112002 (2002).

[36] J. X. Zhang, A. M. Cook, B. J. Munroe, M. A. Shapiro, and R.J. Temkin, Design and high-power testing of a hybrid photonic band-gap (PBG) accelerator structure at $17 \mathrm{GHz}$, in Proceedings of the 6th International Particle Accelerator Conference (JACoW, Richmond, Virginia,
2015), WEPWA062, pp. 2646-2648, http://jacow.org/ IPAC2015/papers/wepwa062.pdf.

[37] A. A. Neuber, M. Butcher, H. Krompholz, L. Hatfield, and M. Kristiansen, The role of outgassing in surface flashover under vacuum, IEEE Trans. Plasma Sci. 28, 1593 (2000).

[38] R. A. Kishek, Y. Y. Lau, L. K. Ang, A. Valfells, and R. M. Gilgenbach, Multipactor discharge on metals and dielectrics: Historical review and recent theories, Phys. Plasmas 5, 2120 (1998).

[39] R. A. Kishek and Y. Y. Lau, Multipactor Discharge on a Dielectric, Phys. Rev. Lett. 80, 193 (1998).

[40] M. N. Buyanova, V.E. Nechaev, and V.E. Semenov, Multipactor discharge on a dielectric surface in the field of circularly polarized plane waves, Radiophys. Quantum Electron. 50, 893 (2007).

[41] O. V. Sinitsyn, G. S. Nusinovich, and T. M. Antonsen, Selfconsistent nonstationary two-dimensional model of multipactor in dielectric-loaded accelerator structures, Phys. Plasmas 16, 073102 (2009).

[42] C. Chang, G. Liu, C. Tang, C. Chen, and J. Fang, Review of recent theories and experiments for improving highpower microwave window breakdown thresholds, Phys. Plasmas 18, 055702 (2011).

[43] J. G. Power, W. Gai, S. H. Gold, A. K. Kinkead, R. Konecny, C. Jing, W. Liu, and Z. Yusof, Observation of Multipactor in an Alumina-Based Dielectric-Loaded Accelerating Structure, Phys. Rev. Lett. 92, 164801 (2004).

[44] C. Jing, W. Gai, J. G. Power, R. Konecny, S. H. Gold, W. Liu, and A. Kinkead, High-power RF tests on X-band dielectric-loaded accelerating structures, IEEE Trans. Plasma Science 33, 1155 (2005).

[45] C. Jing, J. G. Power, R. Konecny, W. Gai, Z. Yusof, S. H. Gold, A. K. Kinkead, V. Dolgashev, and S. G. Tantawi, High power testing of a fused quartz-based dielectric loaded accelerating structure, in Proceedings of the 22nd Particle Accelerator Conference, PAC-2007, Albuquerque, NM (IEEE, New York, 2007), p. 3157, http://accelconf .web.cern.ch/AccelConf/p07/PAPERS/THPMS075.PDF.

[46] C. Jing, W. Gai, J. G. Power, R. Konecny, S. H. Gold, A. K. Kinkead, S. G. Tantawi, V. A. Dolgashev, and A. Kanareykin, Progress toward externally powered $\mathrm{X}$-band dielectric-loaded accelerating structures, IEEE Trans. Plasma Science 38, 1354 (2010).

[47] M. P. Brizhinev, S. V. Golubev, D. S. Dorozhkina, B. G. Eremin, V. G. Zorin, A. G. Litvak, I. V. Plotnikov, S. V. Razin, V. E. Semenov, A. V. Strikovskii, and O. N. Tolkacheva, Microwave discharge on a dielectric surface in vacuum, J. Exp. Theor. Phys. 92, 986 (2001).

[48] A. Neuber, J. Dickens, D. Hemmert, H. Krompholz, L. L. Hatfield, and M. Kristiansen, Window breakdown caused by high-power microwaves, IEEE Trans. Plasma Science 26, 296 (1998).

[49] A. Neuber, D. Hemmert, H. Krompholz, L. Hatfield, and M. Kristiansen, Initiation of high power microwave dielectric interface breakdown, J. Appl. Phys. 86, 1724 (1999). 